\title{
MODELOS DE REGRESIÓN LINEAL PARA ESTIMACIÓN DE TIEMPOS DE VIAJE EN SISTEMAS DE TRANSPORTE MASIVO
}

\section{LINEAL REGRESSION MODELS FOR FORECASTING OF TRAVEL TIME IN BUS RAPID TRANSIT}

Orlando Antonio Sabogal Cardona', Juan David Hincapié Zea², Jhon Jairo Santa Chávez², John Willmer Escobar ${ }^{4}$

Fecha de recepción: 15 de Septiembre de 2014

Fecha de aprobación: 21 de Abril de 2015

Referencia: O.A. Sabogal Cardona, J.D. Hincapié Zea, J.J. Santa Chávez, J.W. Escobar. (2015). Modelos de regresión lineal para estimación de tiempos de viaje en sistemas de transporte masivo. Ciencia e Ingeniería Neogranadina, 25 (1), pp.77- 89

\section{RESUMEN}

Uno de los aspectos más importantes en una implementación de Sistemas de Prioridad (TSP) para el transporte masivo es conocer el tiempo de llegada y de viaje de los buses solicitando prioridad. El presente artículo estima los tiempos de llegada de los buses usando técnicas de regresión lineal para un modelo de simulación del sistema de transporte masivo de la ciudad de Pereira, Colombia. Se presta especial atención a la validez de los supuestos de los modelos lineales en diferentes corredores bajo distintas condiciones. Las simulaciones se han realizado en el software Transmodeler ${ }^{\circledR}$ y los análisis de los modelos usando el lenguaje de programación R. Los resultados muestran que es difícil construir un modelo de regresión lineal válido y que las violaciones a los supuestos de normalidad, independencia y homocedasticidad son frecuentes. La única situación en la que fue posible construir modelos válidos fue en una zona sin intersecciones señalizadas ni estaciones de parada. Sin embargo, evaluaciones a las variables

\footnotetext{
1. Ingeniero Industrial, Joven Investigador, Grupo de Investigación SIRIUS. Universidad Tecnológica de Pereira, Pereira, Colombia, orlando@sirius.utp.edu.co 2. Ingeniero Electrónico, Investigador, Grupo de Investigación SIRIUS. Universidad Tecnológica de Pereira, Pereira, Colombia, judaz@sirius.utp.edu.co

3. Ingeniero Eléctrico, Ph.D. en Ingeniería Eléctrica, Profesor. Universidad Tecnológica de Pereira, Pereira, Colombia, jjsanta@utp.edu.co

4. Ingeniero Industrial, Ph.D. en Operations Research, Profesor. Pontificia Universidad Javeriana Cali, Cali, Colombia, jwescobar@javerianacali.edu.co
} 
de respuesta de tiempos de viajes y análisis de residuales indican que se deben usar modelos lineales generalizados.

Palabras clave: Análisis de regresión, Bus Rapid Transit (BRT), modelos lineales, simulación, Sistemas de Prioridad (TSP).

\section{ABSTRACT}

One of the most important aspects in the implementation of Priority Systems (TSP) in mass transportation is to know the arrival and travel time of the buses requesting priority. This paper estimates the arrival times of buses using linear regression techniques for a simulation model of the mass transportation system of Pereira, Colombia. In particular, special attention to the validity of the assumptions of linear models in different corridors under different conditions is given. The simulations were performed in the Transmodeler ${ }^{\circledR}$ software and the analysis of the models were done using $\mathrm{R}$ programming language. The results show that it is difficult to construct a valid linear regression model. In addition, the violations of the assumptions of normality, independence and homoscedasticity are frequent. The only situation in which it was possible to build valid models was in an area without signalized intersections and stop stations. However, evaluations made to the travel time, response variables, and residual analysis indicate that generalized linear models should be used.

Keywords: Bus Rapid Transit (BRT), Linear Models, Regression Analysis, simulation, Systems Priority (TSP).

\section{INTRODUCCIÓN}

El transporte público eficiente es uno de los grandes desafíos que las ciudades colombianas están enfrentando en la actualidad. Es un tema que afecta directamente a la mayoría de ciudadanos y tiene efectos considerables sobre la economía, el trabajo, la salud y la contaminación. Los sistemas de transporte masivo (Bus Rapid Transit - BRT) se han ido implementado en el país en diferentes ciudades. Desde el año 2000 funciona en Bogotá D.C. un sistema de transporte masivo llamado Transmilenio, el cual fue inspirado en las experiencias previas que se tuvieron en Curitiba, Brasil [1].

Pronto, Transmilenio se volvió un modelo a seguir y un referente estándar de movilidad y alternativa de transporte. Otras ciudades colombianas empezaron a replicar la experiencia de Bogotá D.C. Por esta razón, actualmente hay sistemas de transporte masivo en Cartagena, Bucaramanga, Medellín, Cali y Pereira. Aunque estos sistemas han mejorado la movilidad, los esfuerzos no han sido suficientes. Los BRT y otros modos de transporte público no son eficientes y no son 
capaces de competir con el transporte privado en cuanto a costos, confort, tiempos de viaje y cantidad de pasajeros [2-3].

Esta situación no es exclusiva de las ciudades colombianas, otros países como Estados Unidos, Francia y Nueva Zelanda han experimentado situaciones similares [4], y han usado enfoques de ingeniería centrados en sistemas inteligentes de transporte (ITS por sus siglas en inglés) para contrarrestarlos. Sobresalen entre estos ITS los Sistemas de Prioridad (Transit Signal Priority - TSP), cuya filosofía es priorizar el transporte público en las intersecciones señalizadas. Esto significa cambiar, de acuerdo a unos parámetros y estrategias establecidos, el normal funcionamiento de los semáforos afectando sus fases y ciclos. Las estrategias clásicas de TSP se dividen en dos grupos: estrategias pasivas y estrategias activas.

Las estrategias activas tienen un buen rendimiento pero requieren una implementación tecnológica compleja. Las estrategias activas más comunes son «verde temprano» y «verde extendido». El verde temprano reduce los tiempos de verde en las calles no priorizadas para que los vehículos puedan transitar más rápido. El verde extendido aumenta el tiempo en las fases para que los vehículos puedan pasar por la intersección sin esperar un ciclo completo. Para lograr estas estrategias, primero se necesita hacer la identificación de los buses, que deben ser detectados cuando están cerca de una intersección lo que usualmente se hace con sensores o dispositivos GPS. Después de que un bus es detectado, una señal se envía al sistema de administración de tráfico, si existe, o al respectivo controlador. Por último, la central de administración de tráfico o el controlador deben determinar si una señal de petición es atendida o no, cómo priorizarlas, reducir o extender las fases, establecer tiempos, rotar los ciclos y determinar los tiempos de recuperación. Por esta razón, complejos algoritmos son utilizados en los sistemas TSP para que funcionen en tiempo real.

Las estrategias pasivas son menos costosas y no necesitan de tanta implementación tecnológica, pero son consideradas soluciones malas e imprecisas. Las estrategias pasivas configuran los planes de señalización de tal manera que beneficien el transporte público, por ejemplo, hacer una ola de verde sobre una avenida con transporte masivo o predecir los tiempos de llegada y cambiar las fases con base en en ellos. Las estrategias pasivas no son muy populares y cuando empezaron los TSP fueron subestimadas sin ninguna razón particular. También han surgido estrategias híbridas que combinan conceptos activos y pasivos.

Estados Unidos es uno de los países que más implementaciones TSP ha realizado. Este país tiene amplia literatura en variedad de temas relacionados con TSP. Ova et al. [5] mencionan que la primera implementación TSP documentada fue realizada en Washington en 1962 cuando se hizo un experimento para ajustar los tiempos en un corredor señalizado para aumentar la velocidad de los buses públicos. En este trabajo se analizan cinco implementaciones TSP. Zhang [6] considera implementaciones de TSP en Estados Unidos, Canadá, Inglaterra, Francia y Alemania. En el 2005, el Departamento de Transporte de Estados Unidos publicó un manual para proyectos TSP, Ova et al. [5]. En dicho documento se explican las tecnologías asociadas a los sistemas TSP, estrategias y metodologías para su implementación. También se incluyen algunos casos que revisan todo el proceso de conceptualización, estudios, 
diseños, implementación y puesta en marcha de los sistemas.

Para estudios de factibilidad y de impacto de sistemas TSP clásicamente se han usado técnicas de simulación. En [5-6] se resumen algunos trabajos importantes. Otros trabajos relevantes han sido propuestos por [7-11]. Una pregunta recurrente en proyectos de simulación para TSP y en implementaciones tiene que ver con la cuantificación del beneficio o algunas medidas de efectividad. Marín et al. [3] discuten dicha situación. Otros trabajos han sido propuestos por Garrow et al. [12] y Tan et al. [13].

En la actualidad existen nuevos enfoques para desarrollo de estrategias TSP. Se han hecho esfuerzos para mejorar las predicciones de los tiempos de llegada y combinar TSP con sistemas de control de tráfico dinámico o adaptativo. Liu et al. [14] diseñan un modelo dinámico que mezcla sistemas de control de tráfico adaptativo con TSP. Dion et al. [15] muestran un estudio realizado en Arlington Virginia para incorporar TSP en intersecciones coordinadas. Ambos trabajos concluyen que TSP beneficia el transporte público y no crea problemas al tráfico.

Otro trabajo interesante ha sido propuesto por Tan et al. [16]. En dicho trabajo, dos modelos para predecir tiempos de llegada han sido propuestos. En particular, uno de ellos utiliza regresión lineal para estimar los tiempos de viaje de los vehículos, mientras que el otro se alimenta de los modelos de regresión lineal para diseñar un esquema de filtros de Kalman considerando un enfoque bayesiano.

Para mejorar los sistemas de transporte masivo en Colombia es importante encontrar una alternativa TSP viable y económica. Estrategias mixtas, usando pocas detecciones y predicciones en los tiempos de llegada pueden ser una solución. La estimación de los tiempos de viaje es un factor primordial para minimizar los tiempos de espera de los pasajeros, la optimización del número de paradas de los buses y la determinación de mejores rutas.

En este trabajo se usa el software Transmodeler ${ }^{\circledR}$ para representar un corredor del BRT MEGABUS de la ciudad de Pereira. Con los resultados obtenidos de la simulación, se ha utilizado regresión lineal con las variables de distancia, tiempo y velocidad. Las simulaciones y los modelos se hicieron para diferentes escenarios con el objetivo de probar capacidad del sistema para generar modelos cuando se cambian ciertos parámetros. Los resultados indican que los modelos de regresión no son buenos para predecir los tiempos de llegada porque hay violaciones a los supuestos de normalidad, independencia y homocedasticidad. Los resultados también sugieren que se deben usar modelos lineales generalizados, ya que los modelos residuales y las variables de respuestas se comportan como distribuciones Chi-Cuadrada o Poisson.

\section{MATERIALES Y MÉTODOS}

Para el desarrollo de la investigación se ha usado un caso de estudio del corredor del sistema de transporte masivo MEGABUS del Área Metropolitana Centro Occidente (AMCO) de la ciudad de Pereira. El corredor utilizado es el carril exclusivo que comienza en la Carrera 6.a con Calle 13 a la altura del Viaducto César Gaviria Trujillo y va hasta la Calle 24 con Carrera 7.a en el Parque El Lago Uribe Uribe. A lo largo del corredor hay tres estaciones del sistema masivo y siete intersecciones semaforizadas. El corredor se puede apreciar en la Figura 1. 
La construcción del modelo de simulación propuesto está basada en los lineamientos típicos para esta clase de estudios señalados en [14-15]. Se construyen las vías con los sentidos y giros correspondientes, se agregan los planes de señalización para las intersecciones y se crea el sistema de transporte público con el carril exclusivo y las estaciones. Dentro del modelo se crean los sensores que capturan las variables de velocidad y tiempo de viaje de cada vehículo que pasa por el sensor. En total se han creado 220 sensores identificados con un ID único que va desde el 466 hasta el 686.

Los sensores permiten medir las variables de todos los vehículos durante su recorrido y esa información capturada se usa para estimar en cuánto tiempo va a llegar a una intersección. De esta manera, si es posible hacer una buena estimación de los tiempos de viaje, será posible establecer estrategias de prioridad más eficientes cuando se implemente un sistema TSP.

Para los análisis y la construcción de los modelos se han considerado cuatro subcorredores (ver Tabla 1). El primer subcorredor corresponde al corredor inicial completo. El segundo corresponde a la parte ubicada entre el Viaducto y la Carrera 6.a con Calle 13. El tercero es muy pequeño e incluye una estación, está entre la Carrera 6.a con Calle 13 y Carrera 6.a con Calle 14 y las dos intersecciones que abarca están semaforizadas. El último corredor va de la calle 13 con Carrera 6.a hasta la Calle 19 con Carrera 6.a y no hay ninguna intersección semaforizada.

Tabla 1. Información de los subcorredores.

\begin{tabular}{|c|c|c|c|}
\hline Corredor & Inicio & Fin & Sensor \\
\hline 1 & Viaducto & Calle 25 & $466-686$ \\
\hline 2 & Viaducto & Calle 13 & $466-480$ \\
\hline 3 & Calle 13 & Calle 14 & $483-495$ \\
\hline 4 & Calle 14 & Calle 19 & $497-585$ \\
\hline
\end{tabular}

Se han formulado dos tipos de modelos de regresión lineal para el problema propuesto. El primer modelo usa solamente las mediciones de tiempo de viaje para cada vehículo cuando pasa por cada sensor y toma la ubicación de cada sensor desde el punto de inicio (Viaducto) como una variable de distancia. El objetivo del primer modelo es explicar los tiempos de viaje en función de la distancia. El segundo modelo considera los tiempos de viaje y las medidas de velocidad para tratar de explicar el tiempo de llegada a un sensor (el último sensor de cada subcorredor)

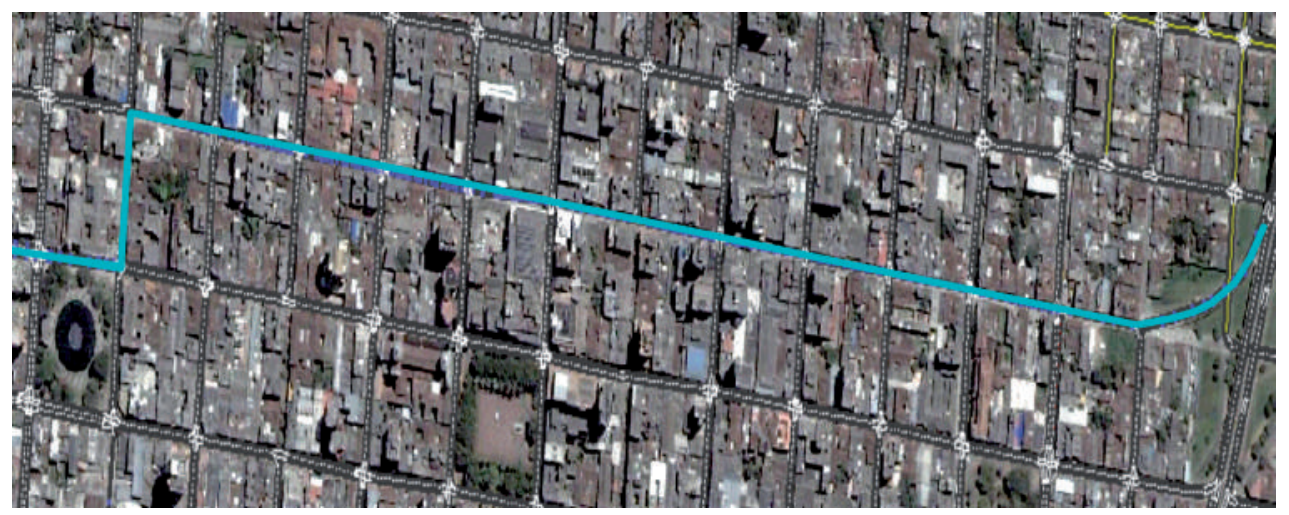

Figura 1. Zona de estudio. 
como función de las variables tiempo de viaje y velocidad en los sensores anteriores.

Ahora bien, el rendimiento de cada modelo puede variar dependiendo del plan de señalización utilizado. Para intentar comprender los efectos que pueden tener los planes de señalización se establecen dos tipos de planes: uno con un ciclo de 60 segundos y tiempo de servicio en verde para el carril exclusivo de 25 segundos, y otro con un tiempo de ciclo de 50 segundos y tiempo de servicio en verde de 20 segundos. Los Ilamaremos nivel alto y nivel bajo respectivamente (Ver Tabla 2). El rendimiento también se puede ver afectado por la frecuencia de partida de los vehículos y por eso de nuevo se manejan dos niveles para esta variable: uno alto con un tiempo de salida promedio de 90 segundos y otro bajo con promedio de salida de 45 segundos (ver Tabla 3).

Emulando el vocabulario y las buenas prácticas del diseño experimental, se tiene un diseño de dos factores con dos variables de 2 niveles cada una (diseño $2^{\wedge} 2$ ). Los modelos de regresión lineal fueron construidos para cada tratamiento en cada corredor. Como se aprecia en la Tabla 4 se tienen 4 tratamientos.

Tabla 2. Niveles de los planes de señalización.

\begin{tabular}{|c|c|c|}
\hline Plan de Señales & Tiempo de ciclo & $\begin{array}{c}\text { Tiempo de } \\
\text { verde }\end{array}$ \\
\hline Alto & 60 & 25 \\
\hline Bajo & 50 & 20 \\
\hline
\end{tabular}

Tabla 3. Niveles de los tiempos de partida

\begin{tabular}{|c|c|c|}
\hline Rata Partida & Tiempo medio & Varianza \\
\hline Alto & 90 & 15 \\
\hline Bajo & 45 & 15 \\
\hline
\end{tabular}

Tabla 4. Experimento $2^{\wedge} 2$ tratamientos

\begin{tabular}{|c|c|c|}
\hline Tratamiento & Plan de señales & Rata partida \\
\hline 1 & Bajo & Alto \\
\hline 2 & Alto & Alto \\
\hline 3 & Bajo & Bajo \\
\hline 4 & Alto & Bajo \\
\hline
\end{tabular}

\section{RESULTADOS}

\subsection{RESULTADOS PARA LOS MODELOS TIEMPO DE VIAJE - DISTANCIA}

Los resultados para los cuatro tratamientos siempre muestran violaciones a los supuestos de normalidad, homocedasticidad e independencia. Las gráficas de tiempo de viaje contra distancia hacen evidente que la varianza no es constante y que incrementa a medida que la distancia recorrida aumenta. Las Figuras 2 y 3 ilustran la situación para diferentes corredores y tratamientos. Para cada figura se ha calculado el respectivo coeficiente de Pearson, obteniendo valores de 0.86 y 0.89 para el primer y segundo tratamiento respectivamente. De esta manera se puede comprobar que el fenómeno que se estudia es efectivamente lineal.

De las gráficas anteriores es claro que la variabilidad de los tiempos de viaje aumenta a medida que los buses avanzan en los recorridos, lo que hace muy difícil hacer estimaciones con precisión. Una explicación para el aumento de la variabilidad es que se van acumulando eventos como paradas en las estaciones, paradas en los semáforos y mayor interacción con otros buses. 


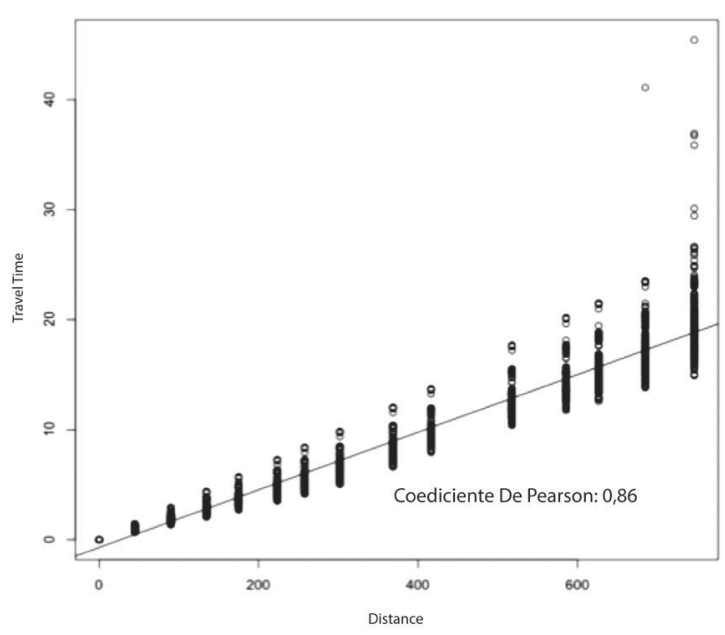

Figura 2. Tiempos de viaje contra Distancia para el segundo corredor en el primer tratamiento.

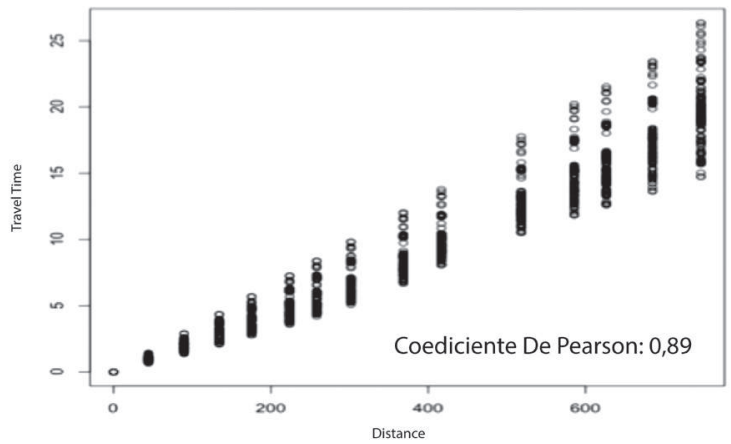

Figura 3. Tiempo de Viaje contra Distancia para el segundo corredor en el cuarto tratamiento.

También se observan las gráficas de residuales para analizar la adecuación del modelo. Las Figuras 4 y 5 muestran gráficas de residuales.
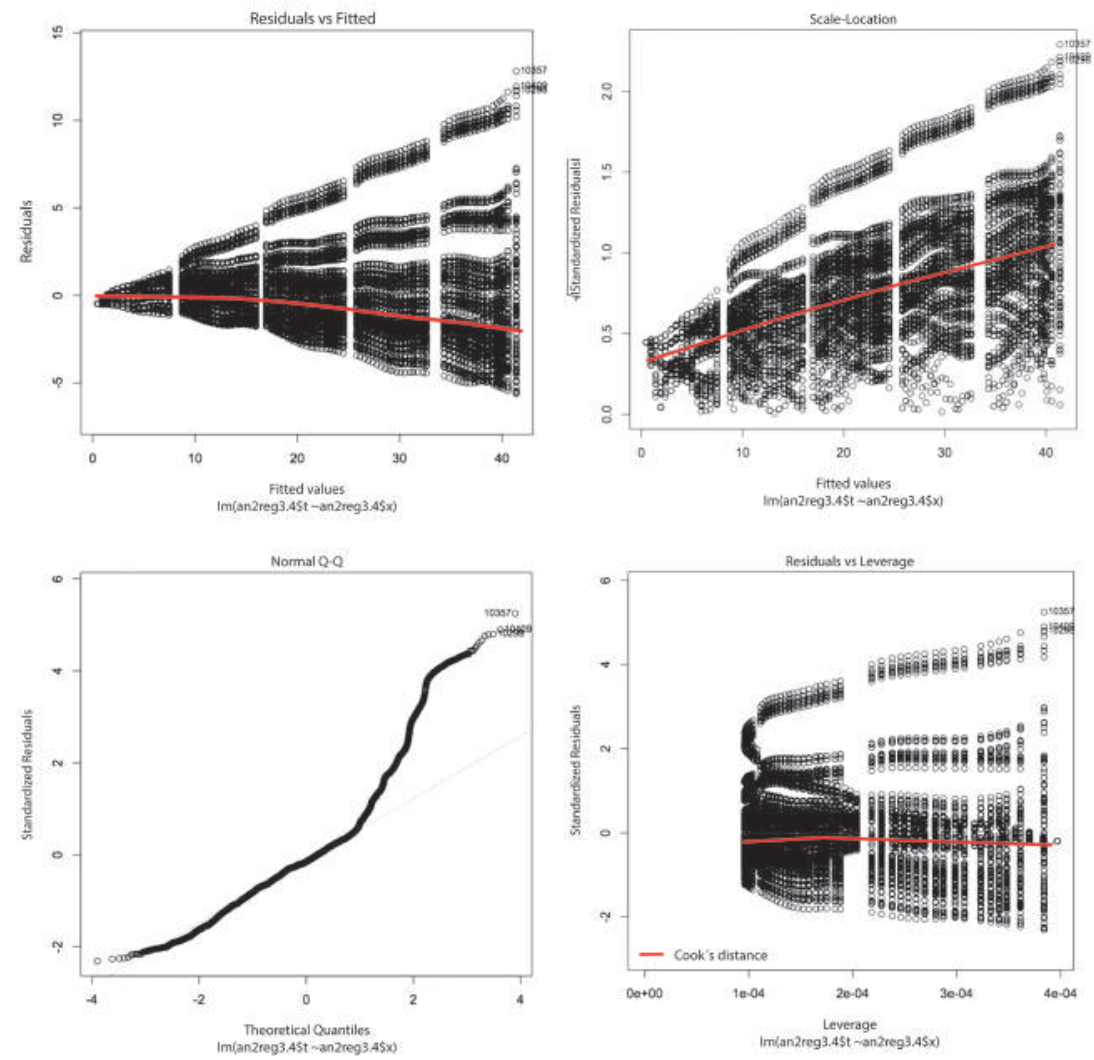

Figura 4. Gráficas de adecuación del modelo y de residuales para el segundo corredor en el primer tratamiento. 

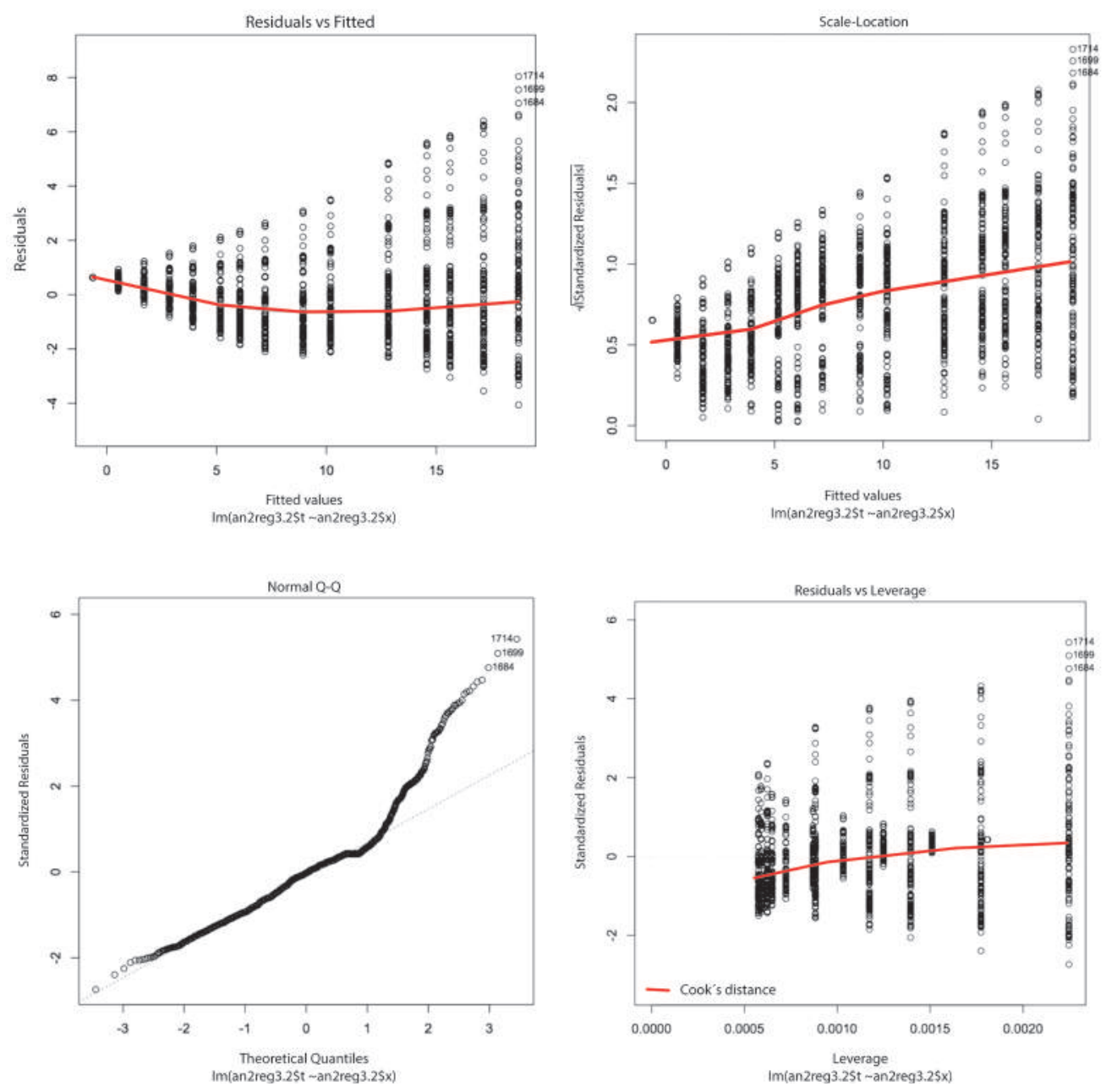

Figura 5. Gráficas de adecuación del modelo y residuales para el cuarto corredor en el tercer tratamiento.

En la Figura 4 es evidente que la variable no tiene varianza constante. En la Figura 5 se observa que la información no sigue la línea recta por lo que no se puede demostrar normalidad. En la Figura 5 se observan algunos patrones y esto evidencia la dependencia entre los datos. En general, la Figura 6 evidencia que no se cumplen los supuestos de un modelo de regresión lineal y no se puede demostrar la adecuación de ningún modelo.
2.2. RESULTADOS PARA LOS MODELOS DE TIEMPO DE VIAJE EN UN SENSOR VELOCIDAD Y TIEMPOS DE VIAJE EN LOS SENSORES ANTERIORES

Los modelos tienen coeficientes $R$ cuadrados y R cuadrados ajustados buenos y se encuentran por encima del $80 \%$. La Figura 6 corresponde a los resultados obtenidos de una regresión lineal en el corredor 2. 
La Figura 7 muestra las gráficas de residuales y adecuación del modelo. En la grafica de la esquina superior izquierda de la Figura 7, se puede apreciar que se detecta varianza no constante del modelo. De la Figura 7 se puede inferir una distribución con una cola en vez de una normal. La Figura 8 muestra un histograma de los residuales confirmando que no se ajustan a una distribución normal.

En el corredor 3, se pudo ajustar un modelo de regresión lineal adecuado. La Figura 9 muestra las gráficas de residuales, normalidad, homocedasticidad e independencia para el corredor 3. La Figura 10 muestra el histograma de residuales. Su comportamiento se asemeja a una distribución normal.

\section{CONCLUSIONES}

En este articulo se han analizado modelos de regresión lineal para estimar los tiempos de viaje en Sistemas de Transporte Masivo.

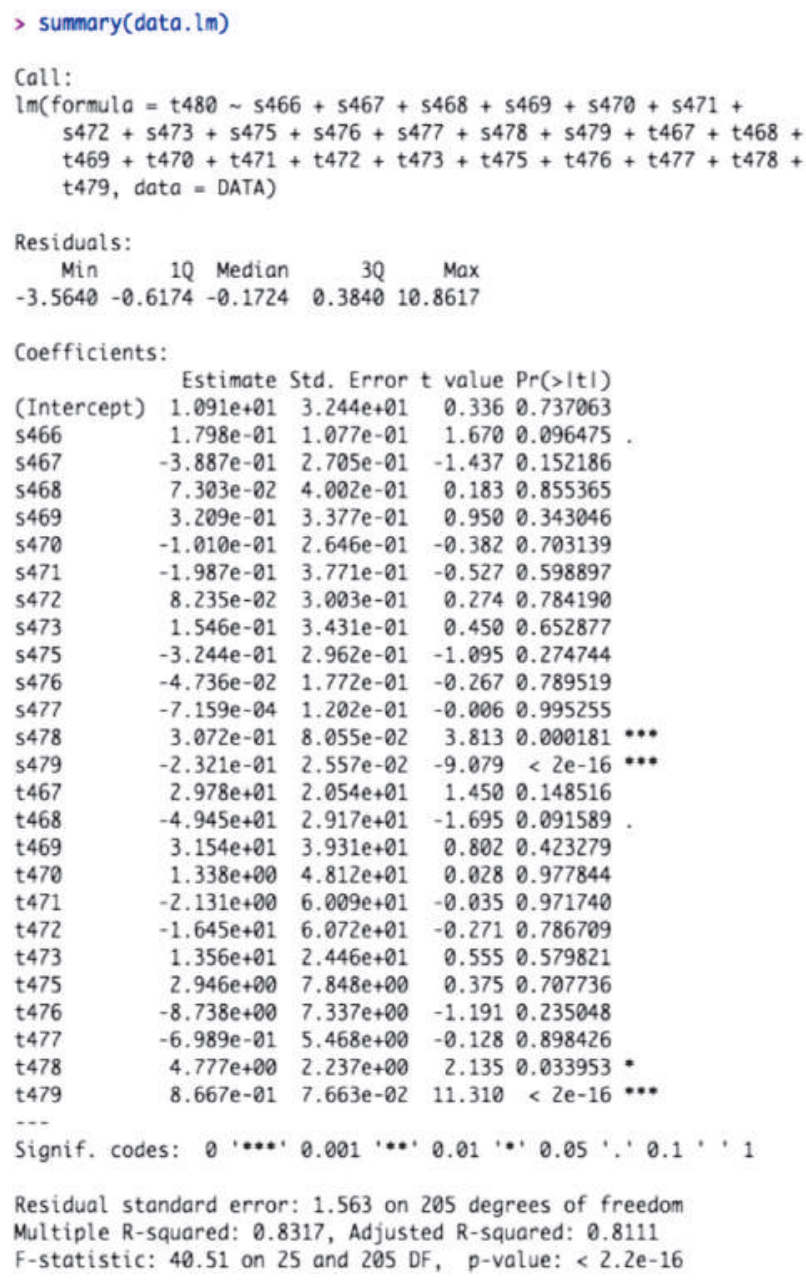

Figura 6. Resumen modelo de regresión lineal en el corredor 2. 

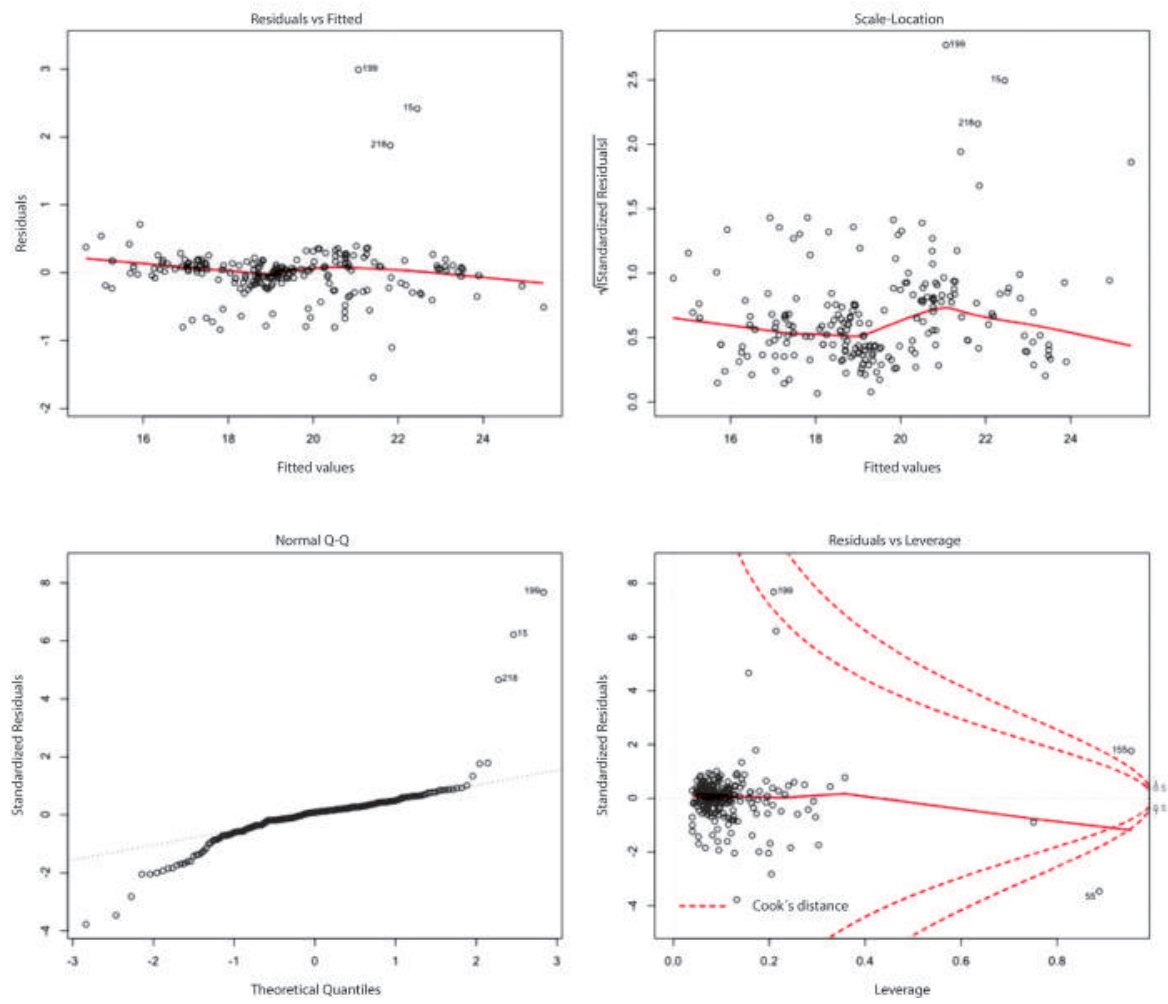

Figura 7. Adecuación del modelo y gráficas de residuales para el segundo corredor.

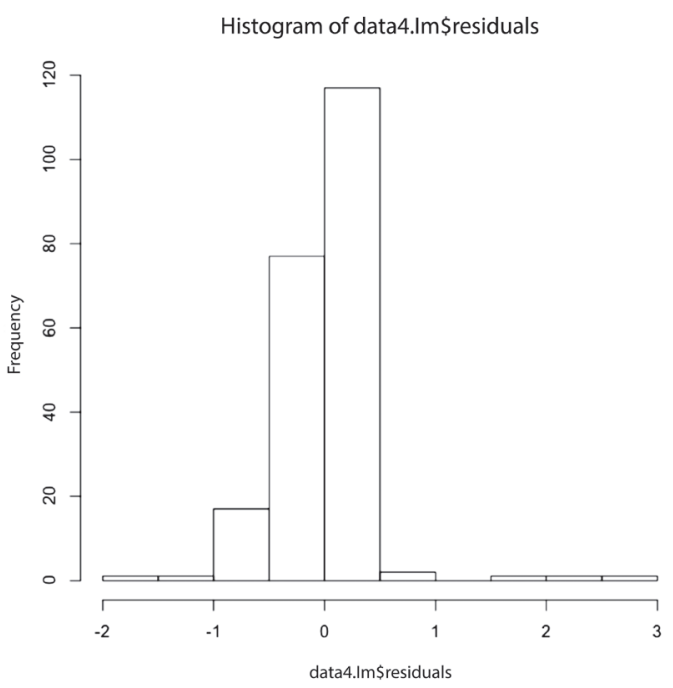

Figura 8. Histograma de residuales en el modelo lineal para el segundo corredor.
Para validar la metodología propuesta se han utilizado datos reales relacionados con un corredor del BRT MEGABUS de la ciudad de Pereira, Colombia.

Para los corredores analizados y los subcorredores se ha determinado que no es factible usar modelos de regresión lineal clásica para estimar los tiempos de viajes. Las violaciones a los supuestos de normalidad, independencia y homocedasticidad son inevitables. Sin embargo, el único corredor en el cual fue posible usar una regresión lineal válida no tenía estaciones de paradas ni intersecciones señalizadas, por lo que se puede pensar que estos dos factores afectan la adecuación de un modelo en escenarios 

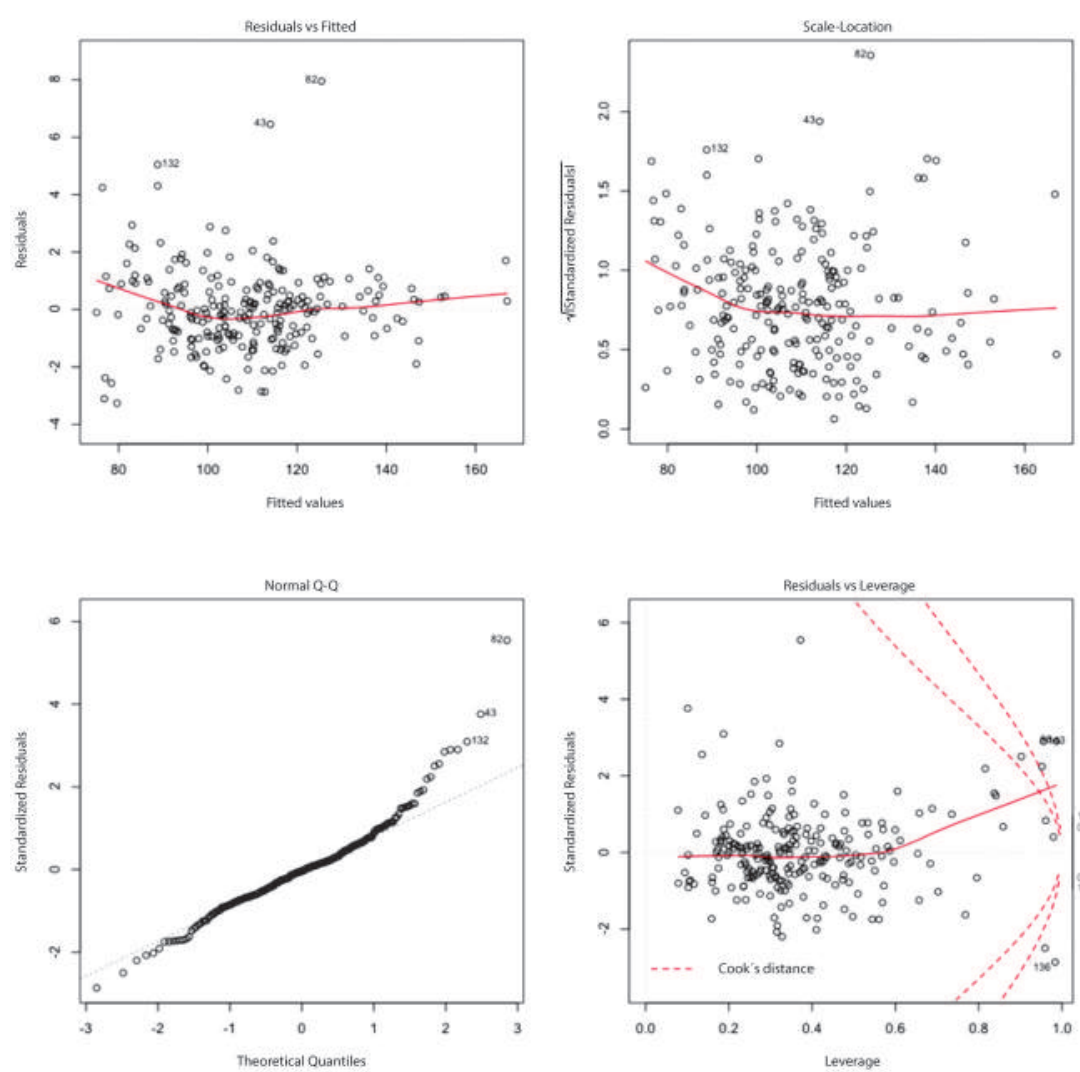

Figura 9. Adecuación del modelo y gráfica de residuales para el modelo lineal en el corredor 3.

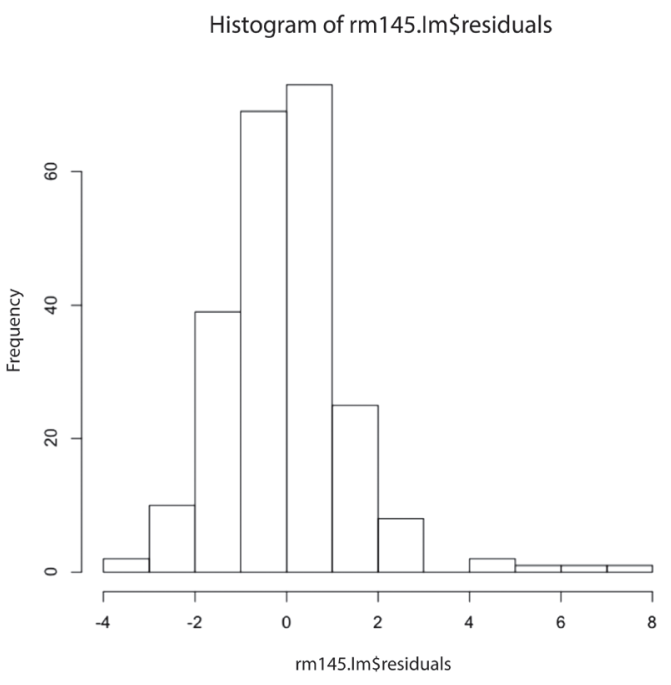

Figura 10. Histograma de residuales reales. Este es un hecho muy importante para considerar en vista de que los sistemas de transporte masivo de Colombia se caracterizan por tener muchas estaciones de parada muy próximas entre sí y estar ubicados sobre carriles con intersecciones señalizadas. Por lo tanto, los modelos para predecir los tiempos de viaje como los propuestos por Dion et al.[15] y Tan et al.[16] no son replicables en los sistemas de transporte masivo colombiano.

Los datos para los modelos de regresión lineal propuestos tienen muchos puntos influenciables que llevan al rechazo de los supuestos. Modelos de regresión robusta y 
múltiple deben ser analizados como trabajos futuros.

El comportamiento de la variable respuesta y los residuales muestran claramente que el fenómeno estudiado no proviene de una distribución normal. El análisis gráfico sugiere que pueden venir de una familia exponencial, especialmente una distribución Chi-Cuadrada - Poisson. Modelos Lineales Generalizados deben ser usados para construir mejores modelos de estimaciones de tiempo de viaje.

\section{AGRADECIMIENTOS}

El primer autor agradece al Gobierno Nacional de Colombia y a COLCIENCIAS por el apoyo en esta investigación a través del programa "Jóvenes Investigadores e Innovadores".

LainvestigaciónhasidoapoyadaporlaUniversidad Tecnológica de Pereira, agradecimientos especiales al Grupo de Investigación Sirius por su apoyo en el soporte técnico y a la Pontificia Universidad Javeriana Cali.

\section{REFERENCIAS}

[1] Lindau, L.A., Hidalgo, D., y Facchini, D. (2010). Curitiba, The Cradle of Bus Rapid Transit. Built Environment, 36(3), 32.

[2] CEPAL. (2008). Los cambios en los Sistemas Integrados de Transporte Masivo (SITM) en Ciudades de América Latina. Boletín Facilitación del Comercio y el Transporte en América Latina y el Caribe, Edición No 259.

[3] Marín, J.A. y Gil, C. (2011). Seguimiento a los SITM. Infraestructura y Desarrollo,
Cámara Colombiana de la Infraestructura, 42. Recuperado de http://www. infraestructura.org.co/revistacci/42/ RevistalD42.pdf.

[4] Gardner, K., D`Souza, C., Hounsell, N., Shrestha, B. y Bretherton, D. (2009). Reviews of Bus Priority at Traffic Signals Around The World. UITP Working Group, Final Report Version 2.0. Recuperado de https://www.tfl.gov.uk/cdn/static/cms/ documents/interaction-of-buses-andsignals-at-road-crossings.pdf.

[5] Ova, K. y Smadi, A. (2001). Evaluation of Transit Signal Priority Strategies for Small-Medium Cities. Recuperado el 5 de junio de 2013 de http://www.ugpti.org/ research/projects.php?view=144.

[6] Zhang, Y. (2001). An Evaluation of Transit Signal Priority and SCOOT Adaptive Signal Control (Tesis de maestría inédita). Virginia Tech, EE.UU. Recuperada el 5 de junio de 2013 de http://oatd.org/oatd/ record?record=oai\% 5C:VTETD\% 5C:e td-05212001-231555.

[7] Dale, J.J., Atherley, R.J., Bauer, T., y Madsen, L.A.(1999) Transit Signal Priority Impact Assessment Methodology Greater Reliance on Simulation. Annual Transportation Research Board Meeting. Washington, D.C., EE.UU.

[8] Davol, A.P. (2001). Modeling of Traffic Signal Control and Transit Signal Priority Strategies in a Microscopic Simulation Laboratory (Tesis de maestría inédita). Massachusetts Institute of Technology, EE.UU. Recuperado de https://its.mit. edu/sites/default/files/documents/ TRAFF.PDF. 
[9] Martin, P.T. Evaluation of Transit Signal Priority Strategies for Bus Rapid Transit on 5600 West Street in Salt Lake County, Utah. (2010). North Dakota State University - Upper Great Plains Transportation Institute, Fargo, EE.UU.: Mountain-Plains Consortium. Recuperado el 02 abril de 2014 de http:// www.ugpti.org/resources/reports/ details.php?id=652 ().

[10] Ekeila, W., Sayed T. y, El Esawey, M. (2009). Development of a Dynamic Transit Signal Priority Strategy. En Annual Conference of the Transportation Association of Canada. Vancouver, British Columbia.

[11] Skabardonis, A., y Christofa, E. (2011). Impact of Transit Signal Priority on Level of Service at Signalized Intersections. Procedia - Social and Behavioral Sciences, 16, 612-619.

[12] Garrow,M.yRandyM.(1997).Development and Evaluation of Transit Signal Priority Strategies. University of Austin, Texas, EE.UU. Recuperado el 2 de abril de 2014 de http://swutc.tamu.edu/publications/ technicalreports/472840-00068-1.pdf ().
[13] Tan, C., Park, S., Liu, H., Xu, Qing., Lau, P. (2008). Prediction of Transit VehicleArrival Time for Signal Priority Control: Algorithm and Performance. IEEE Transactions on Intelligent Transportation Systems, 9(4), 688-696.

[14] Liu, H., Skabardonis, A., y Zhang, W. (2003). A dynamic model for adaptive bus signal priority. TRB Annual Meeting. Washington, D.C., EE.UU. Recuperado de http://trid.trb.org/view. aspx?id=644669.

[15] Dion, F., Rakha, H., Zhang, Y., y Collura, J. (2003). Transit Signal Priority Impacts along a Fixed-Time Signalized Arterial. Intelligent Vehicles Symposium 2003. Proceedings. IEEE, 540, 450-455.

[16] Tan, C., Partners for Adv. Transit \& Highways Program, California Univ., Berkeley, CA, Park, S., Zhou, K., Liu, H., Lau, P., Li, M., y Zhang, W. (2006). Prediction of transit vehicle arrival times at signalised intersections for signal priority control. IEEE Intelligent Transportation Systems Conference (1477-1482). Toronto, Ontario, Canada: IEEE. 